\title{
Establishment of GPS Receiver Test/Calibration Baseline Network in Chongqing
}

\author{
Wenjuan Bai ${ }^{1, \text { a }}$
}

${ }^{1}$ Chongqing Vocational Institute of Engineering, Chongqing, China, 402260

${ }^{\mathrm{a}}$ email

Keywords: GPS receiver, Baseline network, Test, Calibration

\begin{abstract}
In order to ensure a long-term use of GPS receiver and stable data, the GPS receiver is important to regularly test. GPS receiver test/calibration baseline network in Chongqing Vocational Institute of Engineering is the first completed and passed the acceptance by Surveying and Mapping Management Office. This paper introduces the principle of building a network location, combines with the characteristics of the school site. It focuses on the establishment and stability monitoring of the GPS receiver test/calibration baseline network. It has some practical reference value.
\end{abstract}

\section{Introduction}

In recent years, GPS measurement technology in all industry sectors has been rapid development. GPS receivers are widely used to carry out the control survey, engineering survey, geological disaster monitoring and marine surveying and mapping work, which ensure the quality of work and speed up the progress of construction has played an important role. Therefore, the detection of the GPS receiver is important.

Global Positioning System (GPS) measurement procedures and related specifications are specified receiver verification period not exceeding one year. National mapping departments use GPS receiver is required in accordance with the above requirements will be the instrument for inspection. However, the number of existing GPS receiver test/calibration baseline network southwestern region is very limited, to facilitate the detection of the majority of Chongqing mapping user GPS receiver, no GPS receiver detected Chongqing solve the urgent needs of places in Chongqing urgent need to establish a full-fledged GPS receiver baseline network.

A sophisticated GPS receiver test / calibration baseline network is not a short time to complete the outcome, which after the initial design, building, built retest, monitoring, and post-formal acceptance and put into operation, spent a total of nearly three years time.

\section{The Establishment of GPS Baseline Network}

By Chongqing Engineering Vocational entire campus landscape feature, geological conditions and other comprehensive analysis of the situation, with the state of the GPS standard baseline test field construction requirements, the specific location of the final design of the observation pier, design and implementation of network configuration baseline. College of the existing Banan District of Chongqing Shapingba District, and the two campuses, which are not included in the regional faults, folds, plate tectonic activity, geological structure and stability. So the formation of a very good geographical conditions, a complete design of the USBL, short and long baseline.

But before the baseline network design, you must explicitly test field need to meet environmental conditions. Topics include: GPS receiver verification baseline site should be selected in geological formations strong and stable, conducive to long-term preservation, accessibility, ease of construction; each point should be buried into forced centering of the observation pier, there is no strong electromagnetic signal interference around, sight disorders elevation was not more than $15^{\circ}$; calibration point field layout should contain short distance, short distance and long distance, consisting of baseline net shape can be test.

According to the above requirements, combined with campus characteristics, net-shaped design 
is finalized observation pier point and quantity. Pillar total of 10, were forced to the top of the device. Two campuses each observation points are selected from the pier in the roof of a building concrete structure, wherein two in Banan campus, 8 in Shangqiao campus, the specific point distribution is shown in Figure 2-1, 2-2.

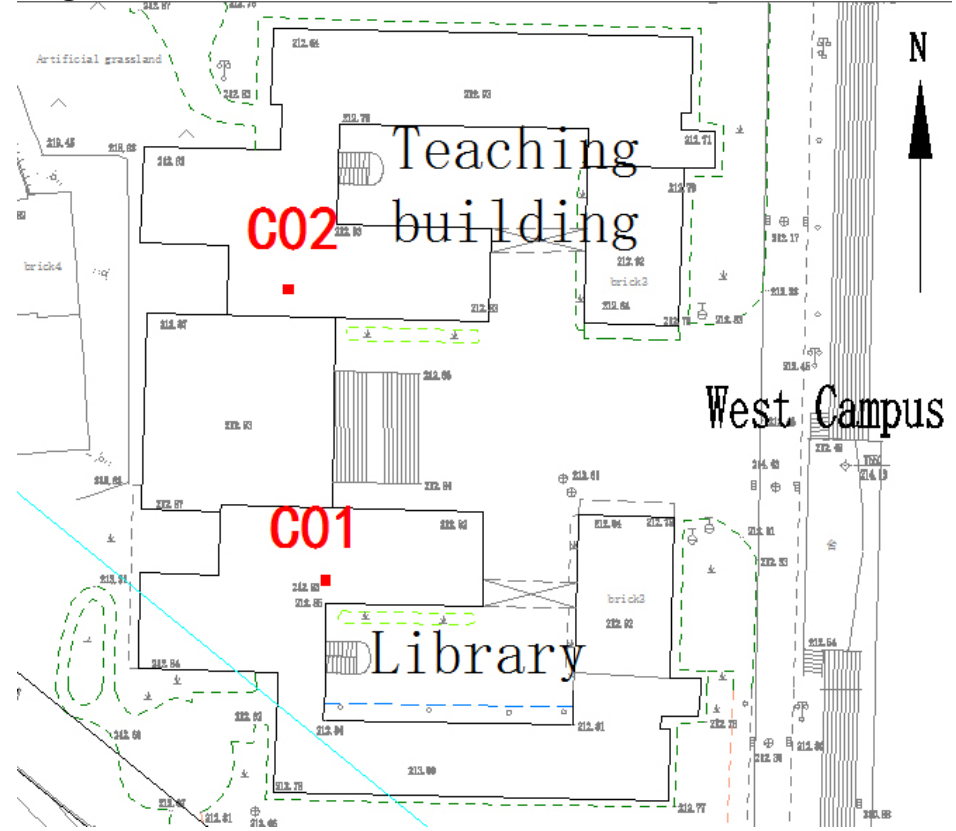

Fig. 2-1 Point distribution map of the Banan campus(C01、C02)

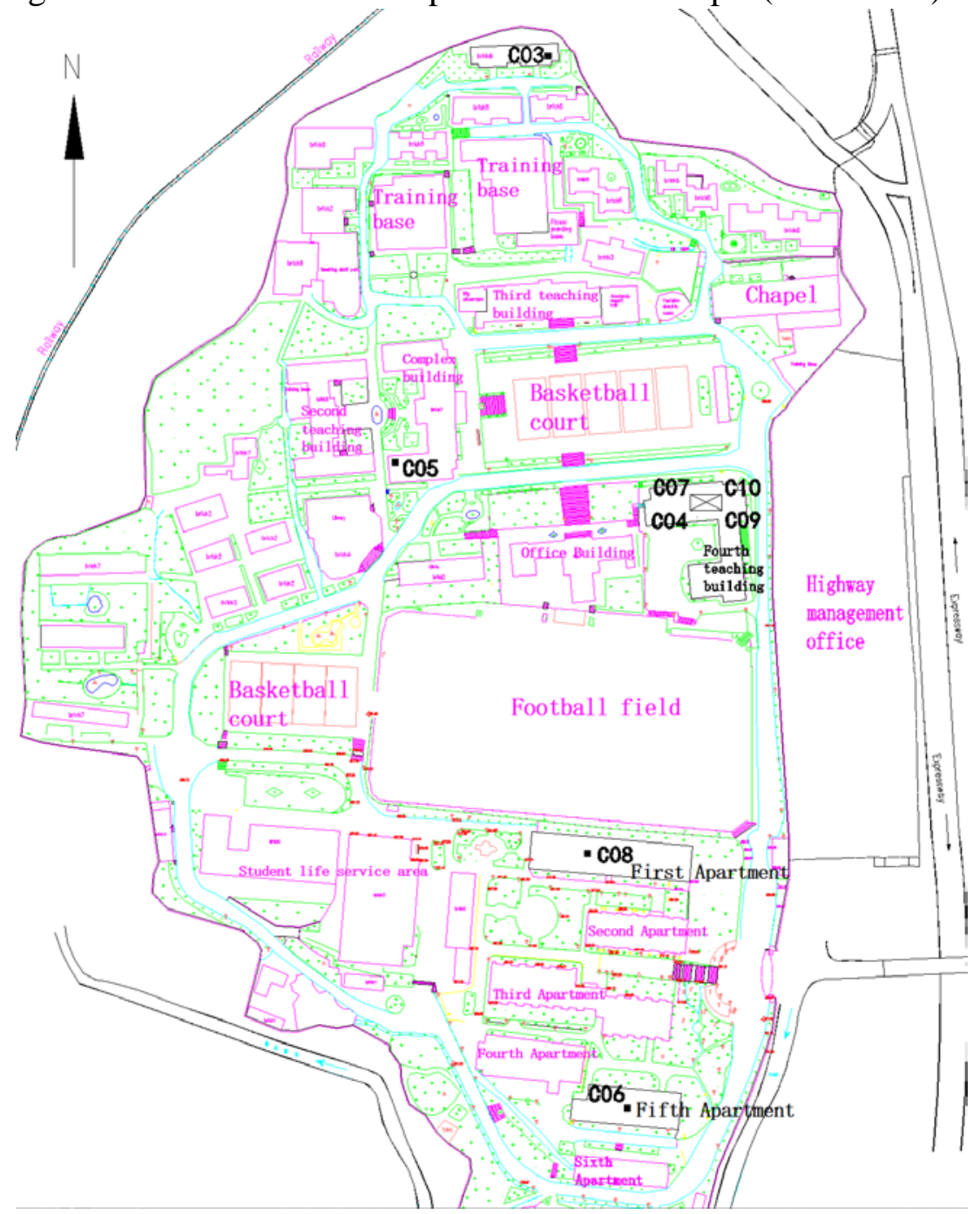

Fig. 2-2 Point distribution map of the Shangqiao campus(C03-C10) 
USBL composed by the four observation pier, observation pier top surface is at the same elevation plane. These observation pier four points in the fourth classroom building roof Chongqing Engineering Vocational College. They will be laid to $5 \mathrm{~m} \times 10 \mathrm{~m}$ rectangular arrangement, the roll call were: C04, C07, C09, C10. Its length distribution: 5.00m Baseline 2: C04 to C07, C09 to C10; 10.00m Baseline 2: C07 to C10, C04 to C09; 11.18m Baseline 2: C07 to C09, C04 to C10.

C04, C07, C09 and C10 constitute USBL network shown in Figure 2-3. These four observations mop set at about $5 \mathrm{~m} \times 10 \mathrm{~m}$ rectangular arrangement of.

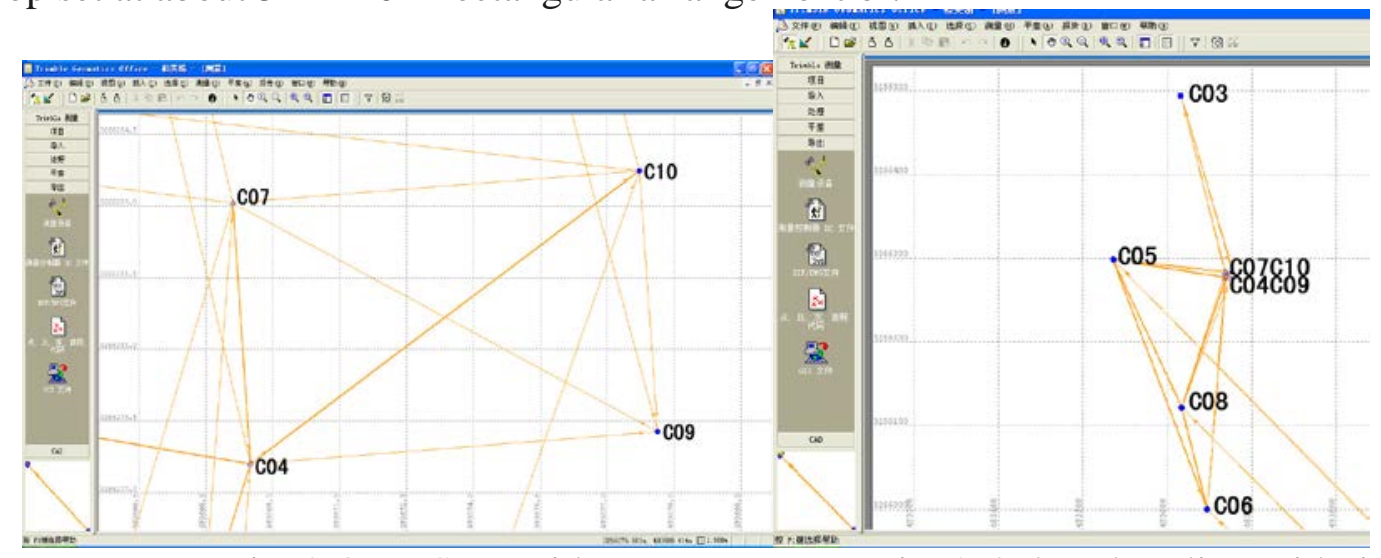

Fig. 2-3 USBL grid

Fig. 2-4 short baseline grid Figure

It consists of eight short baseline observation pier, of which 4 with the USBL's four observation pier total points were distributed in the combination of length between $495 \mathrm{~m} \sim 164 \mathrm{~m}$ baseline distribution. 4 short baseline observation piers were built in families residential buildings (C03) Chongqing Engineering Vocational and Technical College campus on the bridge, Building (C05), a apartment (C08), five apartments (C06) of the roof. Figure 2-4 short baseline grid map.

The above combination USBL observation pier, its length distribution:

C08 and C04, C07, C09, C10 baseline length of about 170m, a total of four; C06 and C04, C07, C09, C10 baseline length of about 280m, a total of four; C05 and C04, C07, C09, C10 baseline length 140m or so, a total of four; C03 and C04, C07, C09, C10 baseline length of about 220m, a total of four; C08 and C03 baseline length of about 370m, a total of 1; C08 and C05 baseline length of about 190m, 1 Article; C08 and C06 baseline length of about 120m, a total of 1; C06 and C05 baseline length of about 310m, a total of 1; C06 and C03 baseline length of about 490m, a total of 1; C05 and C03 baseline length of about 210m, total 1.

8 Pillar 2 Pillar long baseline by the Banan district school campus and on the bridge of the composition, the combined length of $13267 \mathrm{~m} \sim 13679 \mathrm{~m}$ distributed in between. The long baseline observations of two piers were built in the Banan Xiyuan Campus library building (C01) and Teaching Building (C02) of the roof, concrete grid shown in Figure 2-5.

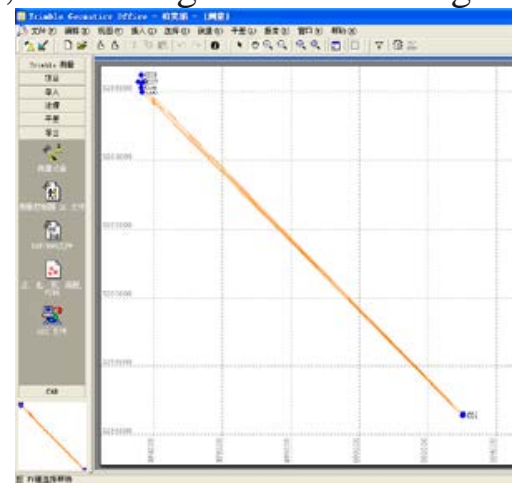

Fig. 2-5 Figure long baseline grid

Chongqing Institute of Engineering Technology GPS baseline network is independent coordinate system. To reduce the length of the deformation plane, keeping GPS coordinates inverse side and the ground side length of the actual measurement consistency, baseline net plane coordinate system uses engineering ellipsoid calculated on the basis of the survey area. The project ellipsoid is defined as: the WGS-84 ellipsoid having the same position, orientation and shape that works ellipsoid space 
Cartesian coordinate system and WGS-84 triaxial coincide, and keeping the first eccentricity (or flat rate) unchanged, but increased the length of the ellipsoid axle, so that the average surface elevation engineering ellipsoid region closest to the baseline network. In this project the ellipsoid using the Transverse Mercator projection, the central meridian of the projection surface selected survey area across the central meridian of the projection baseline survey area in order to weaken due to the length of the projection plane may cause deformation. Projector specific parameters: the central meridian longitude is $106^{\circ} 30$ ', the projection surface of the land height $280 \mathrm{~m}$.

According to technical standards in the relevant specification, in order to ensure GPS test / calibration precision baseline network established to provide reliable baseline data network baseline, baseline network of national norms GPS B-class network. In Field observations and data processing and tolerance requirements, in strict accordance with the relevant provisions of the aforementioned will be administered test.

\section{Baseline Network Stability Monitoring of Chongqing GPS Receiver}

This site for the new network, the observation points are laid on the roof of the new mandatory GPS observation pier, before the completion of the measurement standards and for the community to carry out GPS verification and calibration services, it must be repeated stability monitoring, study its as the feasibility and reliability of measurement standards. Only after all GPS point is considered long-term observations under stable conditions before they can apply for measurement standards certification.

From within one year after the construction is completed, this network was conducted two comprehensive retests. It will coordinate the results of the retest obtained, respectively, with networking and pre-solver achievements by point comparison. Statistical differences coordinate data to determine GPS points and no significant change. Comparative data show, $\mathrm{x}$ direction coordinate difference RMS were $1.1 \mathrm{~mm} 1.2 \mathrm{~mm}$, y direction, and coordinate differences RMS is $2.1 \mathrm{~mm}$ and $1.7 \mathrm{~mm}$, the degree of fit between the two is quite high. Even on (C03 point, the bridge is located on the campus, 101 residential building roof) point of maximum difference, compare the two directions x coordinate differences are $1.7 \mathrm{~mm} 2.8 \mathrm{~mm}$, y coordinate differences and direction and -2.9 , respectively $-2.7 \mathrm{~mm} \mathrm{~mm}$. Therefore, the observation point significant displacement of the entire network did not happen.

In summary, the coordinate differences analysis found no significant change in point of displacement, indicating the point stability is very good. Original GPS network construction and network coordinates retest level difference occurs less than $\pm 5 \mathrm{~mm}$ magnitude of the difference, it is considered mainly due to GPS measurement errors when the original network construction, the measurement error due to retest the joint, the measurement coordinate adjustment the variance value of this small magnitude, it is normal and reasonable.

\section{Conclusion}

Establishing a GPS receiver test and calibration baseline network, not only provides the GPS control survey of students practical training places, training and improving students GPS receiver field observation and inspection instrument capability. At the same time, GPS measurement technology provides a platform for scientific research to improve the theory and practice of teachers GPS measurement technology. The network successfully passed the acceptance and access to the Chongqing Urban Planning Bureau of Surveying and Mapping Management Office approval, provides the community with a GPS receiver testing services.

\section{References}

[1] Wu Lian. GPS receiver calibration Research and Practice [J]. Geospatial information, 2012, 10 (6): 112-115.

[2] Zhang Jie, Zhang Aimin. Jinling GPS receiver for detecting a field study [J]. Modern Surveying 
and Mapping, 2012, 35 (1): 27-29.

[3] Zhang Hui, Zhang Jian. Current Situation and Development Strategy mapping equipment testing market [J]. Industrial Measurement, 2011, 21 (3): 46-47.

[4] Gu Jianxiang, Gao Chao. Establishment and Measurement Uncertainty Shanghai GPS calibration baseline site assessment [J]. City Survey, 2010 (1): 41-44.

[5] Zhu Lijiang, Chen Zhongxin. GPS receiver detection technology based on CORS system[J]. Surveying Engineering, 2010, 19 (5): 35-37.

[6] Zhang Jian, Zhang Hui. Perfect GPS receiver testing services state key construction projects [J]. China Metrology, 2010 (9): 19-21.

[7] Wu Zhiyong. Precision GPS receiver, a long baseline test evaluation[J]. Surveying and Mapping, 2009 (7): 67-69.

[8] Bo Wanju, Huang Liren.. GNSS field test field [J]. Surveying and Mapping, 2009 (4): 48-50.

[9] Cheng Longfei. Anhui GPS receiver design verification field analysis[J]. Science and Technology Information, 2008 (19): 88.

[10] Li Xiaojun. Principles and Methods of GPS receiver verification[J]. Geospatial Information, 2008 (10): 50-52.

[11] Zhang Bigui, Peng Bin. GPS receiver Verification System[J]. Surveying and Mapping, 2005 (10): 28-29.

[12] Zhang Dongming. GPS receiver performance testing and analysis[J]. Kunming Metallurgy College, 2004,20 (1): 4-7.

[13] Wang Guanru. Inspection and maintenance of the Global Positioning System (GPS) receiver[J]. Shanxi mapping, 2003 (4): 43-44.

[14] Zhou Zuyuan. GPS receiver testing, use and maintenance[J]. Mineral mapping, 2002, 18 (1): 30-31.

[15] Zhang Wei. Liaoning. Province to establish a GPS receiver verification field[J]. Heilongjiang Institute of Technology, 2002, 16 (1): 19-22.

[16] Chen Yiqun, Liu Dajie. One kind of test and calculation method of GPS receiver antenna phase center deviation [J]. Surveying and Mapping, 2000 (12): 15-16. 